\title{
A computational approach for design rationalization in Enterprise Architecture
}

\author{
Georgios Plataniotis, Henderik A. Proper \\ Public Research Centre Henri Tudor, \\ Luxembourg, Luxembourg \\ Radboud University Nijmegen, \\ Nijmegen, the Netherlands \\ EE-Team, Luxembourg, Luxembourg \\ \{georgios.plataniotis, erik.proper\}@ tudor.lu
}

\author{
Sybren de Kinderen \\ University of Luxembourg, Luxembourg, \\ Luxembourg \\ EE-Team, Luxembourg, Luxembourg \\ sybren.dekinderen@uni.lu
}

\begin{abstract}
We aim for rationalizing Enterprise Architecture (EA) languages, showing not only final EA designs, but also the reasoning behind these designs (in terms of selection criteria, design alternatives, and more).

Our earlier work proposes the EA Anamnesis approach for architectural rationalization. Its major contribution is a formal metamodel and a corresponding concrete syntax to interrelate business and IT decisions. Yet, up to now, EA Anamnesis lacks software tool support.

As a response, this paper introduces a software tool for EA Anamnesis. In doing so, we contribute (1) a computational assessment for the EA Anamnesis metamodel and a corresponding visual syntax, showing its implementability, (2) a reflection of our aim to develop a tool by rapid prototyping, whereby practitioner feedback enables concurrent maturation of the software tool and metamodel, and (3) the idea of presenting a tool to foster acceptance and practical uptake of EA Anamnesis.
\end{abstract}

Keywords-Enterprise Architecture, Design Rationale, Prototype implementation, Computational assessment

\section{INTRODUCTION}

Enterprise Architecture (EA) modeling languages, prominently the Open Group standard ArchiMate [1], can be used to express the complete "business-to-IT" stack (cf. [2]) of an organization. Particularly, for enterprise designs expressed in such EA languages one can interrelate IT infrastructure, applications, business processes, products/services provided to end customers, and organizational goals [3], [4]. While EA modeling languages allow to model an enterprise holistically, the design decisions behind the resulting models are often left implicit.

In our earlier work [5], [6], [7] we introduced the EA Anamnesis approach for architectural rationalization. EA Anamnesis derives from the ancient greek word $\alpha \nu a ́ \mu \nu \eta \sigma \iota \varsigma$ (/ænæm'ni:sis/), which denotes memory and repair of forgetfulness. EA Anamnesis captures decision characteristics such as decision criteria and used decision making strategy, and shows the relation between business-level and IT-level decisions. Furthermore, EA Anamnesis allows for a formal linkage

The Enterprise Engineering Team (EE-Team) is a collaboration between Public Research Centre Henri Tudor, Radboud University and HAN University of Applied Sciences (www. ee-team.eu) to metamodel-based EA artifacts, thus allowing for a bridge between languages for EA design (prominently ArchiMate) and the corresponding design rationale.

Thusfar, EA Anamnesis lacks software tool support. EA Anamnesis consists of a formal metamodel only supplemented by a way of using it. Yet, such a tool support is relevant (1) to provide for a computational assessment of EA Anamnesis. This means that we should provide evidence that the metamodel and corresponding concrete syntax (see Sect. II-B) can indeed be implemented in a software tool. (2) to foster the practical uptake of a modeling language.

As a response, this paper presents a computational assessment of the EA Anamnesis approach by means of its implementation into a software prototype. We focus our discussion on the prototype implementation, in terms of our tool development by rapid application development, and in terms of lessons learned. For an elaborate discussion of the implemented metamodel see [5], [6], [7]. This paper is structured as follows: Section II discusses tool functionality, implementation aims, and illustrates its use. Section III concludes.

\section{PROTOTYPE IMPLEMENTATION}

This section discusses our prototype development and development aims (in Sect. II-A). Furthermore, in Sect. II-B we briefly illustrate tool usage.

\section{A. Prototype development and aims}

Software tool functionality. We implemented the EA Anamnesis metamodel and the visualization environment which enable us to create visual instantiations of the metamodel in a software tool. Fig. 1 presents such a visual instantiation. This tool conforms exactly to our metamodel. No elements have been added, modified, or removed.

Our software tool allows architects to rationalize architectures through a Graphical User Interface, in accordance with the EA Anamnesis metamodel.

Objectives and development environment. We have three key aims for developing tool support: (1) to provide for a computational assessment of EA Anamnesis. Here, we aim at testing to what extent the metamodel and corresponding 


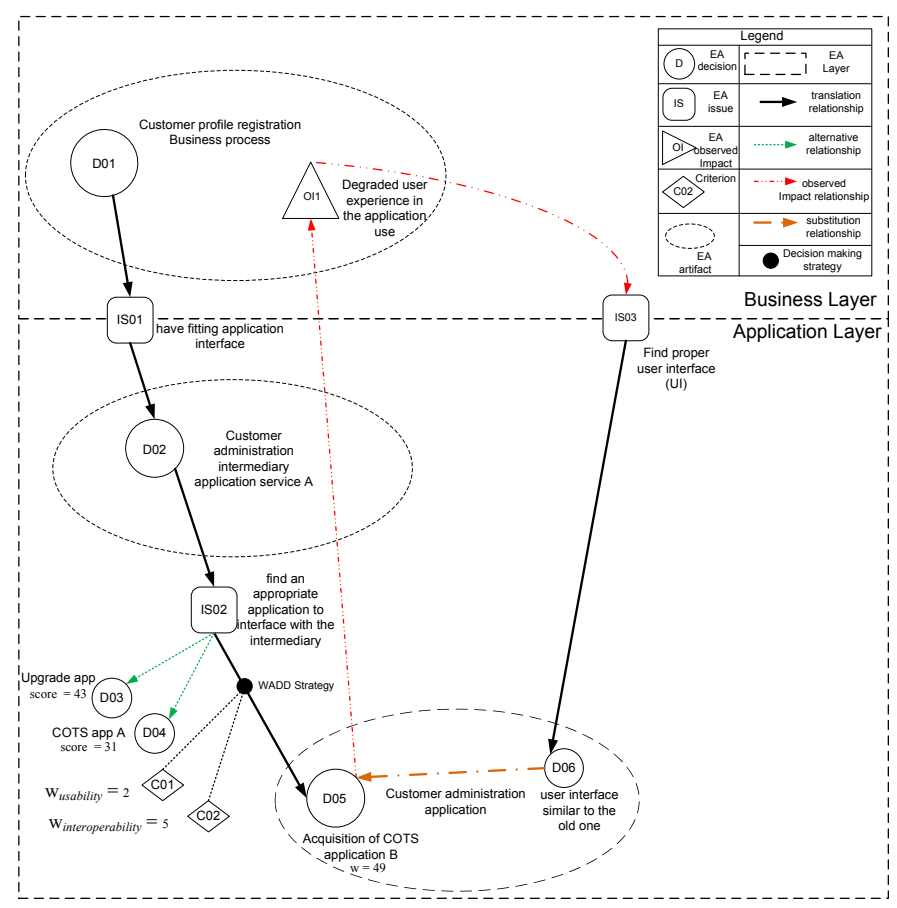

Fig. 1. Prototype tool visualization

concrete syntax can indeed be implemented in a software tool. (2) to showcase (rudimentary) software tool support to practitioners as a means to demonstrate implementability of EA Anamnesis. We consider this relevant since tool support fosters the practical uptake of a modeling language [8]. (3) to process practitioner feedback for further tool development, during upcoming practical validation. Here, we aim at showing the tool as a proof-of-concept during case studies, so that practitioners can react to the presented tool support in terms of, for example, usefulness of EA Anamnesis concepts, and missing concepts and/or functionality. Subsequently, practitioner feedback can be processed concurrently in the metamodel and software tool.

\section{B. Illustrative tooling scenario}

Let us assume that an Enterprise Architect wants to understand the Enterprise Architecture of the organization by examining the visualization of Fig. 1. For the sake of example, we concentrate on the properties and different relationships of EA decision "Acquisition of COTS application B" (D05).We consider two translation relationships visualized by the tool: (1) between "have fitting application interface" (IS01) and "Customer administration intermediary application service A" (D02), and (2) between "find an appropriate application to interface with the intermediary" (IS02) and "Acquisition of COTS application B" (D05). These translation relationships reflect how the requirements for a new business process were translated in the application layer to concrete decisions by John.

Furthermore, the tool visualizes the decision making process for the decision "Acquisition of COTS application B" (D05) as well as the rejected alternatives. As we can see "Acquisition of COTS application B" (D05) was selected among the alternatives "Upgrade app" (D03) and "COTS app A" (D04) because of its highest score ( $\mathrm{w}=121)$ during the evaluation process. Furthermore, the tool represents the observed impact "Degraded user experience in the application use" (0I1) of EA Decision "Acquisition of COTS application B" (D05). We can now observe that users of the customer administration application experienced difficulties to use the new application system. Thus, we see that the EA Decision "Acquisition of COTS application B" (D05) had a negative observed impact on the business process "Customer profile registration". Moreover, we can observe that the observed impact "Degraded user experience in the application use" (0I1) triggered a new issue "Find proper user interface (UI)" (IS03). In order to address the application layer issue "Find proper user interface (UI)" (IS03) John made a new decision "User interface similar to the old one" (D06) in the application layer which is visualized by a translation relationship. EA Decision "User interface similar to the old one" (D06) substitutes Decision "Acquisition of COTS application B" (D05). This is represented by a substitution relationship.

\section{CONCLUSIONS AND FUTURE WORK}

In this paper we presented a computational assessment of the EA Anamnesis approach for architectural rationalization. The computational assessment showed implementability of our approach. Furthermore, we discussed how our choice for rapid prototyping will enable us to concurrently mature the metamodel and software tool during an upcoming evaluation of EA anamnesis with EA practitioners.

\section{ACKNOWLEDGMENTS.}

This work has been partially sponsored by the Fonds National de la Recherche Luxembourg (www. fnr.lu), via the PEARL programme.

\section{REFERENCES}

[1] The Open Group, ArchiMate 2.0 Specification. Van Haren Publishing, 2012.

[2] R. Winter and R. Fischer, "Essential layers, artifacts, and dependencies of enterprise architecture," Journal of Enterprise Architecture-May, pp. 1-12, 2007.

[3] M. Op't Land, E. Proper, M. Waage, J. Cloo, and C. Steghuis, Enterprise architecture: creating value by informed governance. Springer, 2008.

[4] J. Hoogervorst, "Enterprise architecture: Enabling integration, agility and change," International Journal of Cooperative Information Systems, vol. 13, no. 03, pp. 213-233, 2004.

[5] G. Plataniotis, S. de Kinderen, and H. A. Proper, "Capturing decision making strategies in enterprise architecture - a viewpoint," in Enterprise, Business-Process and Information Systems Modeling, ser. Lecture Notes in Business Information Processing. Springer Berlin Heidelberg, 2013, vol. 147, pp. 339-353. [Online]. Available: http://dx.doi.org/10.1007/978-3-642-38484-4_24

[6] G. Plataniotis, S. d. Kinderen, and H. A. Proper, "Relating decisions in enterprise architecture using decision design graphs," in Proceedings of the 17th IEEE International Enterprise Distributed Object Computing Conference (EDOC), 2013.

[7] G. Plataniotis, S. de Kinderen, and H. A. Proper, "Ea anamnesis: An approach for decision making analysis in enterprise architecture," International Journal of Information System Modeling and Design (IJISMD), to appear.

[8] I. Malavolta, P. Lago, H. Muccini, P. Pelliccione, and A. Tang, "What industry needs from architectural languages: A survey," Software Engineering, IEEE Transactions on, vol. 39, no. 6, pp. 869-891, 2013. 